\title{
Téoros
}

Revue de recherche en tourisme

\section{L’assurance qualité}

\section{Un outil à implanter dans le domaine du tourisme}

\section{Normand Dulude et Simon Bastien}

Volume 17, numéro 3, automne 1998

Pratiques contemporaines de gestion dans le domaine du tourisme

URI : https://id.erudit.org/iderudit/1072179ar

DOI : https://doi.org/10.7202/1072179ar

Aller au sommaire du numéro

Éditeur(s)

Université du Québec à Montréal

ISSN

0712-8657 (imprimé)

1923-2705 (numérique)

Découvrir la revue

Citer cet article

Dulude, N. \& Bastien, S. (1998). L'assurance qualité : un outil à implanter dans le domaine du tourisme. Téoros, 17(3), 51-53. https://doi.org/10.7202/1072179ar d'utilisation que vous pouvez consulter en ligne.

https://apropos.erudit.org/fr/usagers/politique-dutilisation/ 


\section{Normand Dulude et Simon Bastien}

D'une manière générale, la qualité est devenue un facteur clé dans la décision d'achat. Dans tous les domaines - etc'est particulièrement vrai dans le domaine du tourisme la concurrence s'est accrue, sollicitant du même coup le client avec une diversité de plus en plus grande de produits et de services.

Miser sur la qualité est la stratégie gagnante par excellence. Cette stratégie, déjà implantée dans bien des secteurs économiques et dans des entreprises performantes, est à toutes fins utiles inexistante dans le domaine du tourisme. L'implantation éventuelle d'un programme qualité dans l'industrie touristique québécoise devrait, selon nous, tenir compte du produit touristique, mais d'abord du service, car c'est ce qui permet de se distinguer.

La notion de qualité dont il est ici question se réfère donc à cette notion de service. Dans une telle orientation, il est envisageable de développer un programme d'assurance qualité qui pourrait s'appliquer à l'ensemble de l'industrie touristique, les éléments de ce programme qualité étant liés directement ou indirectement au service et non au produit comme tel. Commun à l'industrie, nous pensons que le programme qualité à implanter pourrait servir à établir une image de marque autant pour l'entreprise elle-même que pour le Québec en tant que destination touristique d'excellence au plan international.

\section{DES CONCEPTS LIÉS À LA QUALITÉ}

Telle que définie dans les normes internationales, la qualitê d'un produit ou d'un service est son aptitude à satisfaire les attentes explicites et implicites du client. Cela implique de définir clairement ses clientèles cibles, ainsi que leurs attentes et leurs besoins, de façon à préciser le niveau de produit ou de service attendu.

La qualité, c'est aussi la capacité de reproduire le produit ou le service de façon uniforme, toujours au même niveau, d'en garantir la conformité et la constance. L'objectif est de réduire l'écart entre le produit ou le service offert et le niveau recherché par la clientèle. Pour y arriver, on doit s'appuyer sur des normes.

Plus le service offert comporte de lieux, d'intermédiaires, de services et de produits différents, plus le risque de non-conformité est grand. À l'opposé, plus la qualité repose sur le comportement des personnes, plus on a de chances de garantir la conformité. Un guichet automatique a rarement des sautes d'humeur ! Dans tous les cas, cependant, l'objectif est de réduire l'écart entre le service offert et celui recherché par la clientèle.

Le tourisme est davantage une affaire de services qu'une affaire de produits. Le service est l'ensemble des prestations auxquelles le client s'attend, au-delà du produit. C'est particulièrement le cas dans le tourisme. La qualité y dépasse la différence entre le service attendu et le service obtenu ; elle se rapporte aussi à la qualité de l'expérience touristique. La qualité dans les services touristiques devrait être définie comme la recherche de l'excellence dans l'ensemble des éléments nécessaires pour fournir aux visiteurs une expérience touristique plaisante et gratifiante ${ }^{1}$.

La recherche de l'excellence se réfère à la fonctionnalité (qualité du service en soi) et au plaisir (qualité de l'expérience) du service ou d'un ensemble de services. On parlera également des dimensions quantitatives et qualitatives de la qualité.

Parmi l'ensemble des définitions des caractéristiques de la qualité que nous avons trouvées dans l'intéressant compte rendu du congrès 1997 de $1^{\prime}$ AIEST portant précisément sur la gestion de la qualité dans le domaine du tourisme, nous retenons les éléments suivants :

Fiabilité. Récurrence, respect des promesses faites. Vitesse de réaction. Réponse rapide aux besoins et aux demandes du client. Compétence. Compétences et connaissances nécessaires pour offrir le service. Accessibilité. Heures d'affaires, localisation, moyens d'accès (personnes handicapées, fax, etc.). Courtoisie. Politesse, accueil, considerration. Communication. Pouvoir communiquer avec le client dans une langue qu'il connaît. Crédibilité et honnêteté. Démontrer que l'entreprise tient à cour les intérêts de sa clientèle. Sécurité. Installations et équipements, confidentialité. Connaissance des attentes du client.Attributs tangibles. Installations physiques, services disponibles, apparence du personnel, équipements et outils promotionnels. 
Une dếmarche qualité, définie comme un programme d'amélioration continue de la qualité, vise à produire et à maintenir la qualité du service offert. Ce n'est qu'une fois cette maîtrise acquise que l'on peut penser à une forme de reconnaissance de cette qualité. La reconnaissance, quel que soit le modèle privilégié, est l'aboutissement de la démarche qualité.

Il existe divers modèles de reconnaissance :

La certification. C'est l'acte par lequel est affirmé le constat que le produit ou le service livré est conforme à une norme. Elle suppose que l'on se soit entendu sur la norme ou le standard de qualité. La norme est généralement de niveau élevé, de façon à ce que la certification ait un sens et procure une reconnaissance enviable, significative et utile commercialement (par exemple, les normes ISO 9000). La certification a pour objectif de $\propto$ garantir $m$ au client la qualité du produit ou du service offert ou, du moins, de minimiser les risques de non-qualité, et ne peut être accordée sans un contrôle externe préalable. Toute vérification (enquête, examen, contrôle) externe en vue d'une certification est effectué par un organisme indépendant.

Le label de qualité. La labellisation est l'acte par lequel un organisme affirme que ses membres respectent un règlement qui impose souvent un niveau de qualité et d'autres exigences professionnelles (par exemple, un code d'éthique), ce qui les distingue des autres entreprises du même secteur.

Contrairement à la certification qui est établie en référence à une norme échappant à la profession ou à un secteur, la labellisation est établie sous contrôle des membres, qui définissent le niveau de qualité qu'ils souhaitent atteindre, garantir et promouvoir. Elle est pratique courante dans l'industrie touristique, notamment dans les chaînes et les réseaux franchisés.

Les chartes de qualité. Elles sont généralement élaborées par des associations sectorielles ou régionales et sont la plupart du temps transitoires, dans la mesure où elles ne constituent qu'une forme d'engagement sans système rigoureux d'évaluation et de contrôle.
La classification. Sans être un modèle de reconnaissance, la classification est considérée généralement comme une étape ou une partie d'un programme qualité. Celleci a pour objectif d'informer le client de ce quil l'attend en matière d'offre de produit ou de service. De plus, un système de classification efficace amène chez l'exploication. Il est important de souligner que la classification concerne généralement les aspects quantitatifs et matériels de l'offre.

\section{LA QUALITÉ DANS LE DOMAINE TOURISTIQUE : L'ENTREPRISE VS LA DESTINATION}

S'il peut etre facile ( ? !) pour une entreprise de gérer la qualité de ses propres services, il est beaucoup plus difficile de gérer (et de garantir) la qualité d'une destination. De fait, l'entreprise touristique seule n'est pas en mesure de garantir la qualité du a produit * touristique tout entier. Elle peut contrôler uniquement la qualité de sa propre entreprise et de ses propres services. Parler de garantir la qualité d'une destination touristique, comme le Québec, ou de l'une ou l'autre de ses régions ou de ses localités, implique des efforts considérables compte tenu du nombre d'intervenants concernés. Le problème, c'est que la qualité de la destination, perçue par le client, se réfère souvent au maillon faible de la chaîne. tant une volonté d'améliorer sa classifi-

Les efforts foumis par une entreprise touristique pour améliorer sa qualité sont partiellement dépendants des efforts de ses partenaires. Dans un domaine comme le tourisme, où le choix d"une destination se détermine en grande partie par le bouche à oreille, l'appréciation globale de la destination est cruciale. La qualité d'une destination concerne de multiples entreprises et organismes.

Pour qu'une destination puisse atteindre le niveau de qualité recherché, il faut :

- Se donner un critère commun de qualité : la satisfaction du client (et s'entendre sur ce que cela veut dire),

- Se donner des objectifs communs,

- Mettre au point un programme d'action commun (même s'il peut être appliqué sectoriellement),

- Accepter que les bénéfices puissent être inégaux ${ }^{2}$.

Les programmes qualité applicables à une destination ont pratiquement toujours été initiés et portés par les pouvoirs publics, ce qui ne semble pas être un gage de succès, compte tenu de la tendance à s'estomper (et à disparaître) une fois le financement disparu.

Les problèmes des programmes qualité à l'échelle d'une destination tournent, semble-t-il, autour des éléments suivants :

- L'absence ou la déficience de la culture de la qualité au sein des entreprises et

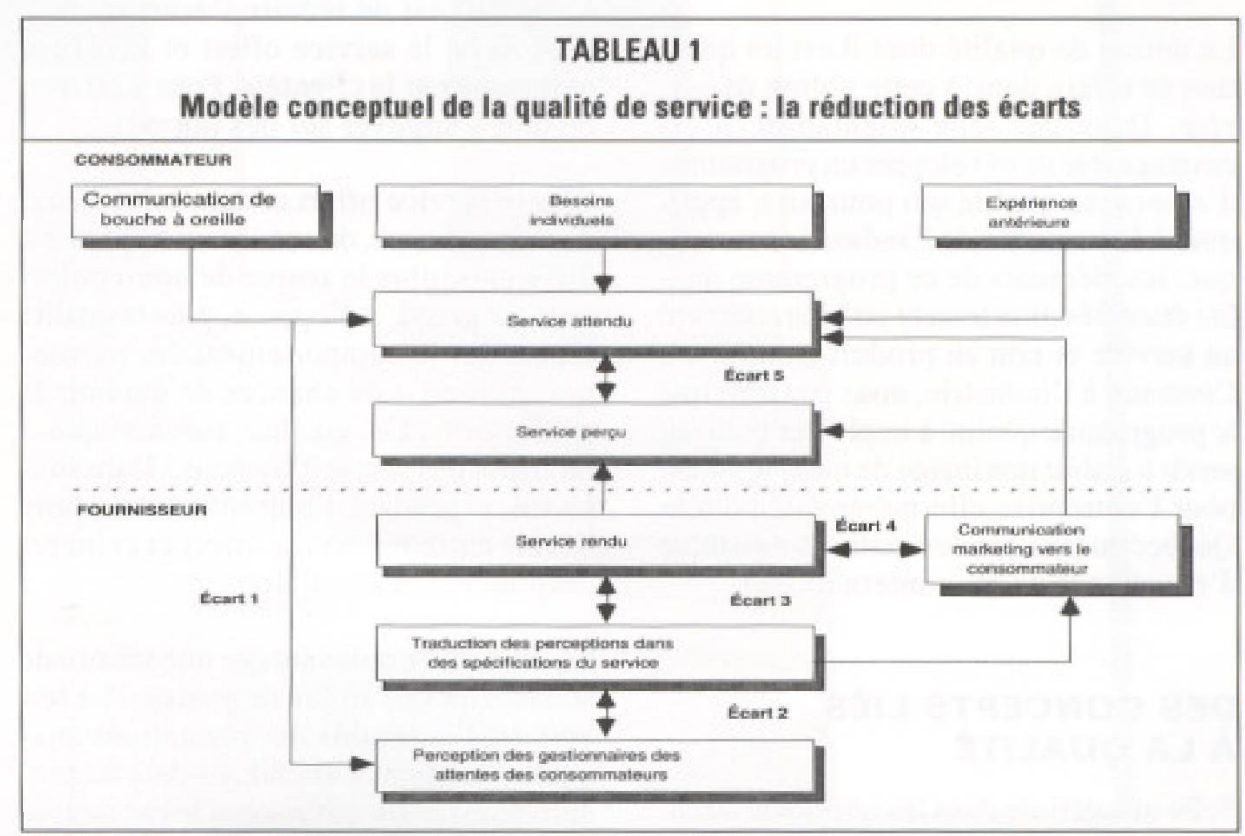

Source ; Berry, Parasuraman, Zeithaml, Ouality Service, Free Press, New York 
des organismes concernés (la qualité est souvent perçue comme la stratégie de dernier recours quand on a le dos au mur),

- L'organisation de la démarche qualité fait défaut,

- Le prix de la qualité passe mal chez certains.

Compte tenu de cela, nous pensons qu'il y a intérêt au Québec à concentrer les efforts sur l'implantation d'un programme qualité au sein des entreprises et non pas en fonction d'une ou de destinations.

\section{LES EFFETS CONSTATÉS D'UN PROGRAMME QUALITÉ}

\section{AU PLAN POSITIF}

Les entreprises qui ont implanté un programme qualité et qui ont été certifiées ont constaté plusieurs effets bénéfiques dont voici les principaux :

- L'amélioration des façons de faire (processus) et la clarification des responsabilités.

- Une meilleure structuration du travail et de meilleurs outils de gestion.

- Une responsabilisation accrue des employés, un plus haut niveau d'autonomic dans la prise de décisions.

- Un sentiment d'appartenance et de fierté renforcé, particulièrement lorsque la certification est obtenue.

- Une diminution des coûts de non-qualité (erreurs, plaintes, délais, etc.) et, par conséquent, une meilleure rentabilité.

- La fidélisation accrue de la clientèle, qui entraîne des besoins promotionnels moindres.

- L'amélioration de l'image de marque de l'entreprise.

- L'acceptation par le client de payer pour la qualité.

Ces effets ont été constatés dans tous les secteurs d'activités où un programme qualité a été implanté, tant dans les entreprises manufacturières que dans les entreprises de services. C'est le cas notamment chez quelques entreprises hôtelières qui ont entrepris et obtenu récemment leur certification ISO.

\section{AU PLAN NÉGATIF}

Les problèmes les plus largement constatés dans l'implantation d'un programme qualité concernent :

- L'absence (ou la déficience) de la culture de la qualité au sein de l'entreprise : on ne s'entend pas sur ce que cela veut dire.

- Le prix de la qualité passe mal : une dépense et non un investissement.

- Les différents modèles de programme qualité sont peu adaptés aux petites entreprises.

\section{AUTRES CONSTATS}

- La qualité de l'accueil pèse deux fois plus lourd que tous les autres aspects dans l'évaluation de la qualité par le client.

- Le personnel est l'outil principal du service touristique.

- Un programme qualité n'a d'avenir que s'il comprend une dimension de contrôle.

- Les programmes qualité s 'implantent plus facilement dans des nouvelles entreprises.

\section{DES * COMPARABLES *}

Nos recherches sur l'implantation de programmes qualité dans l'industrie du tourisme à travers le monde nous amènent à conclure que plusieurs des programmes qualité identifiés vont dans le sens de l'adaptation des normes ISO 9000 au contexte de l'industrie du tourisme. Cela peut s'expliquer par la nécessité qu'un programme qualité en tourisme se rapproche des normes reconnues internationalement. D'ailleurs, on discute actuellement de la possibilité de créer une norme internationale « ISO Tourisme .

Nous pensons qu'un programme qualité à implanter au Québec devrait aller dans le mềme sens, c'est-à-dire s'inspirer des normes ISO tout en tenant compte des particularités de l'industrie québécoise du tourisme et de la situation des entreprises de ce secteur.

\section{DES ORIENTATIONS DE BASE D'UN PROGRAMME QUALITÉ}

Les orientations à la base du programme qualité devraient, selon nous, être les suivantes :

- Un programme qualité pour l'ensemble de l'industrie touristique au Québec et non un programme par secteur ou sous-secteur de l'industrie.

- Un programme qui évalue les aspects matériels, humains et marketing de l'entreprise.

- Un programme qui évalue les processus (façons de faire) plutồ que le produit ou le service lui-mểme.

- Un programme applicable tant aux petites qu'aux grandes entreprises.

- Un programme qui mène à une certification, laquelle ne doit pas être obtenue trop facilement si elle veut garder sa valeur distinctive.

- Un programme évolutif, c'est-à-dire qui peut être implanté par étape.

- Un programme se rapprochant des normes internationales.

- Un programme facultatif et volontaire.

L'implantation d'un programme qualite pour l'industrie touristique québécoise est un défi majeur qui exige des changements importants au cour même de la culture d'entreprise. Nous pensons qu'il s'agit là d'un défi que les entreprises du domaine du tourisme n'ont pas le choix de relever si elles veulent demeurer - ou devenir concurrentielles et faire du Québec une destination de calibre international.

Normand Dulude est président et associé du Groupe DBSF ; Simon Bastien y est directeur de projets.

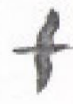

\section{NOTES}

1 Adapté de Ritchie et Crouch (1997), Congrès de l'AIEST.

2 Origet du Cluzeau (1997), La qualité à l’échelon de la destination touristique, AIEST. 